\title{
Medication Errors (MEs) in Several Countries: A Systematic Review
}

\section{Sugeng Wigiyantoro ${ }^{1}$ and Ede Surya Darmawan²}

${ }^{1}$ Student of Hospital Administration Master Programme, Faculty of Public Health, Universitas Indonesia, Depok, West Java, Indonesia

${ }^{2}$ Scientific Lecture, Chairman of Health Administration and Policy Department, Faculty of Public Health, University of Indonesia, Depok, West Java, Indonesia

\section{Abstract}

The increasing number of reports of Medication Errors (MEs) and the subsequent relevant damage, especially in the medical centres, have become a growing concern for patient safety in recent decades. Patient safety, particularly drug safety, is a major concern and the challenge for healthcare professionals around the world. Knowing the prevalence of MEs presented descriptively based on existing studies to approach systematic review. In first stages, journals were searched by using the online

Corresponding Author: Ede Surya Darmawan edesurya@ui.ac.id

Received: 17 October 2018 Accepted: 5 November 2018 Published: 5 December 2018

Publishing services provided by Knowledge E

(c) Sugeng Wigiyantoro and Ede Surya Darmawan. This article is distributed under the terms of the Creative Commons

Attribution License, which permits unrestricted use and redistribution provided that the original author and source are credited.

Selection and Peer-review under the responsibility of the and ICHA Conference Committee.

\section{G OPEN ACCESS} databases through Science Direct and EBSCO. The search used specific keywords such as 'medication errors' and 'administration errors'. As a result, this study reports about Medication Errors in several countries, including two largest hospital of Isfahan in Iran, University Teaching Hospital in Korea, Mansoura Teaching University Hospital, two cases reported in Bolak Eldakror Hospital in Egypt and Midnapore Medical College and Hospital in India. Medication Errors can be prevented by various strategies, such as implementing the six rights of medication administrations principle (right patient, right medication, right dosage, right route of administration, right time and right documentation) and as a control by using a 'double-check' or even 'triple check' procedures before giving the drug to the patient.

Keywords: Medication Errors (MEs), drug administration, medication administration principles

\section{Introduction}

The U.S. Institute of Medicine (IOM) published a report in 1999 by the title of 'To Err Is Human' which medical errors mentioned as a problem of epidemic causing nearly 44.000 to 98.000 deaths annually [1]. This report was remarkable for World Health Organization (WHO) and eventually leading to an announcement prioritizing patient safety as an imperative for health care policy makers around the world [2]. 
Medication errors in hospitals is a major interest to patients because of the central role of medications in treating illness and the important potential consequences to the patient if this is done incorrectly [3].

A medication error is any preventable event that may cause or lead to inappropriate medication use or patient harm while the medication is in the control of the health care professional, patient, or consumer. Such events may be related to professional practice, health care products, procedures, and systems, including prescribing, order communication, product labeling, packaging, and nomenclature, compounding, dispensing, distribution, administration, education, monitoring, and use. [4]

\section{Methods}

The studies undertaken on medication errors were systematically reviewed in November 2016. The exact electronic search strategy is outlined in Figure 1. The search strategy included human studies of all languages, and all types of trials on patients of all ages (refer to PRISMA diagram).

This study uses systematic review method through an online database facility in collaboration with the University of Indonesia: Science Direct and EBSCO. The systematic review using two search keywords 'Medication Errors' AND 'Administration Errors' produced 2,832 results. Furthermore, the screening in the publication of the last five years (2012 to 2016), the content type (journal), subject or topic of the articles (medication errors, drug administration, medication administration), full text or open access journals, and last screened by the inclusion of abstract leaved eight journals. After in-depth review, seven journals are in accordance with the criteria as shown on Figure 1 and table 1.

\section{Results}

The definition of medication errors used on this study is any inconsistency, whether in dosage form, dose, administration route, dosing interval, between what has been ordered by a physician and what the patient received from the nurse. Probable errors are including unauthorized dose, extra dose, under dose, omission dose, wrong dose, wrong route, wrong time and frequency [5]. 

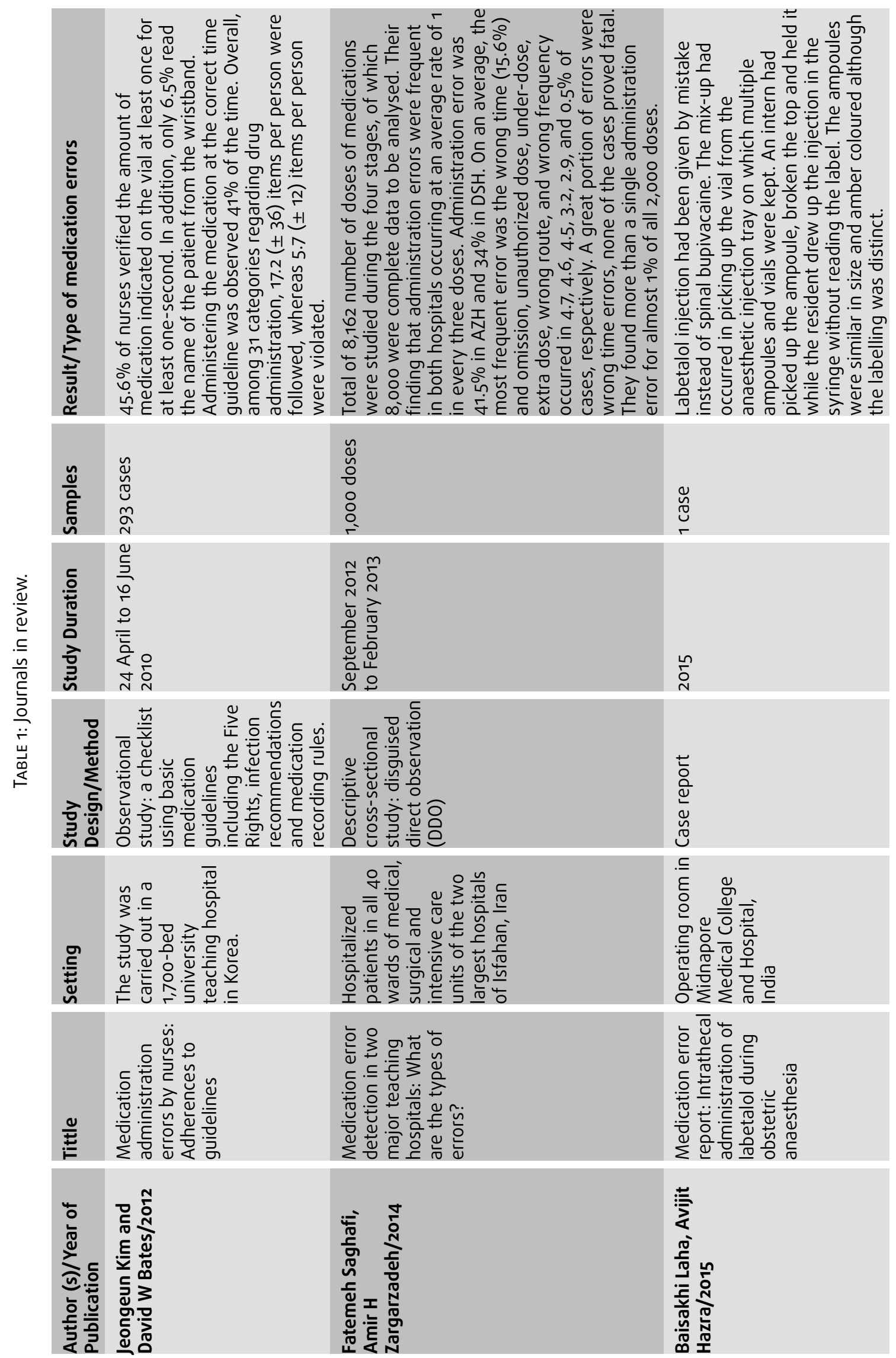


\begin{tabular}{|c|c|c|c|c|}
\hline 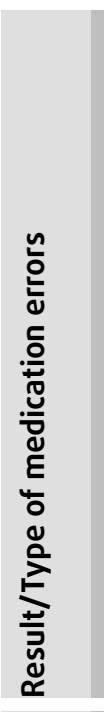 & 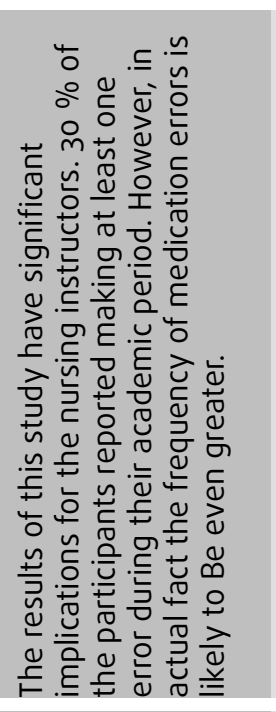 & 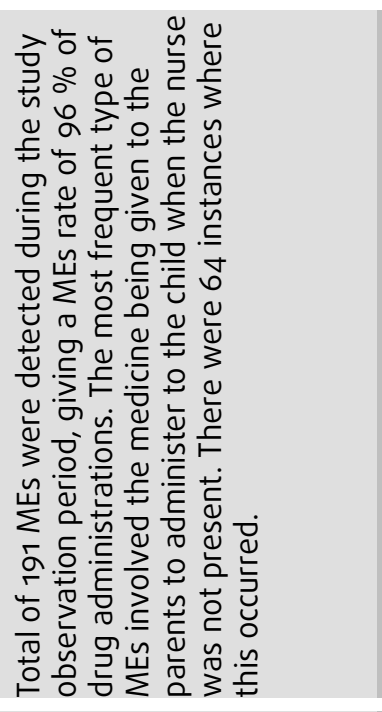 & 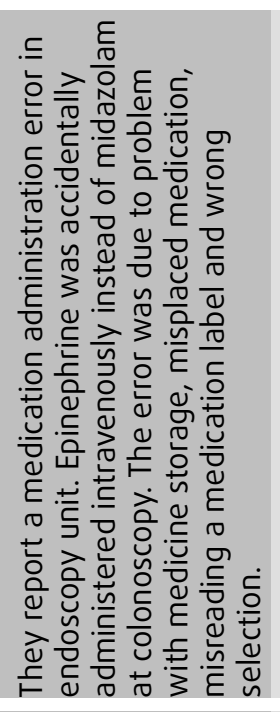 & 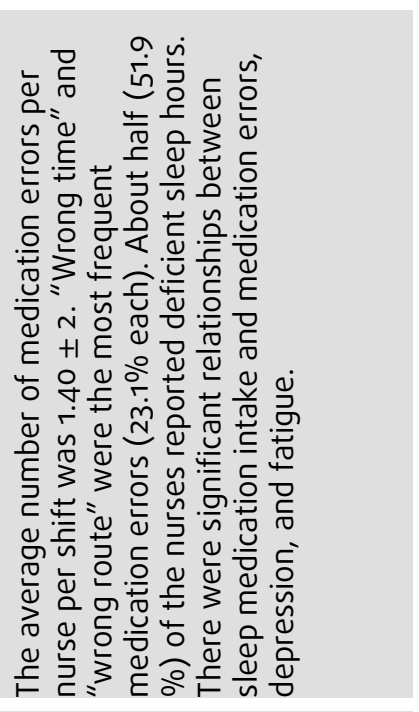 \\
\hline$\frac{\tilde{u}}{\frac{\tilde{a}}{\tilde{E}}}$ & 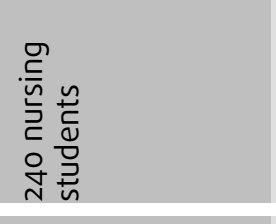 & $\begin{array}{l}\breve{u} \\
\tilde{o} \\
0 \\
\circ \\
o \\
i\end{array}$ & 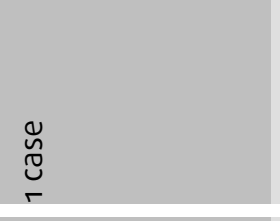 & 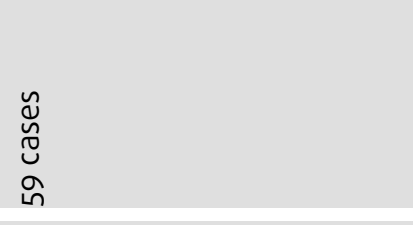 \\
\hline 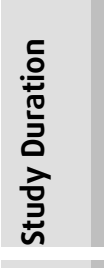 & 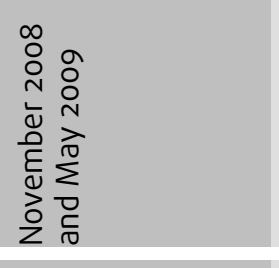 & 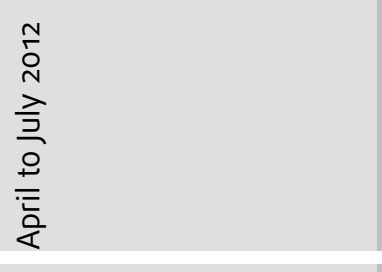 & $\stackrel{N}{N}$ & \\
\hline 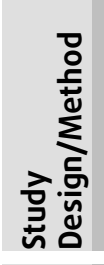 & 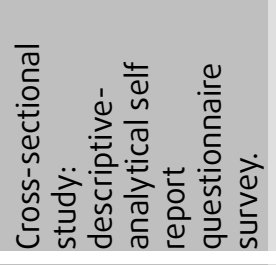 & 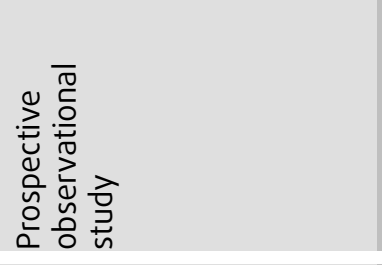 & 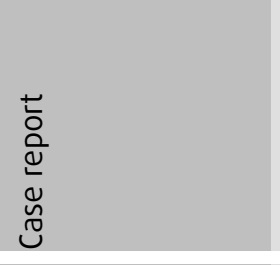 & 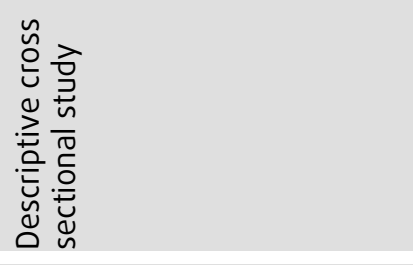 \\
\hline . & 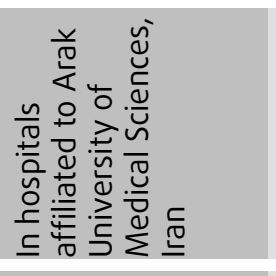 & 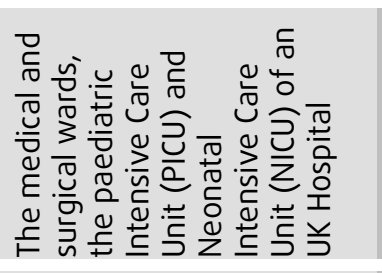 & 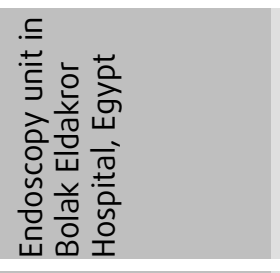 & 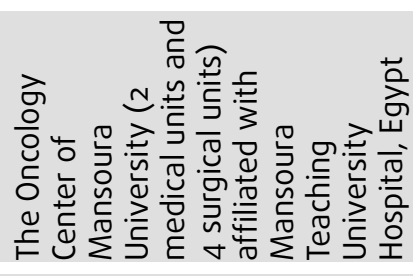 \\
\hline 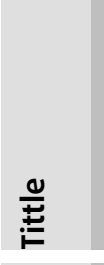 & 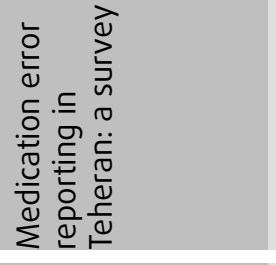 & 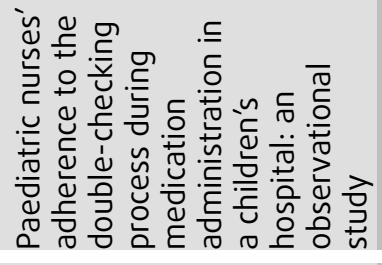 & 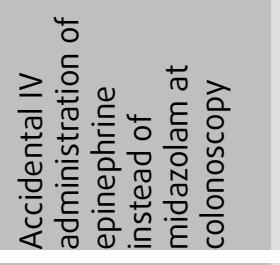 & 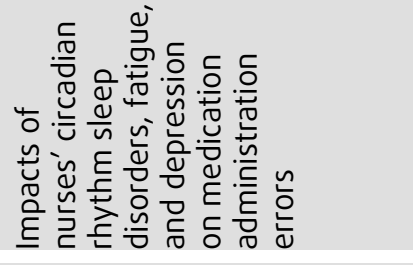 \\
\hline 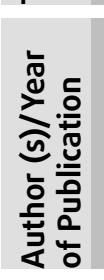 & 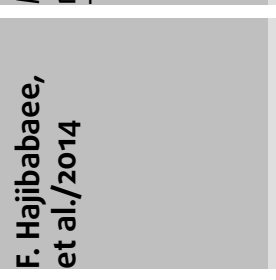 & 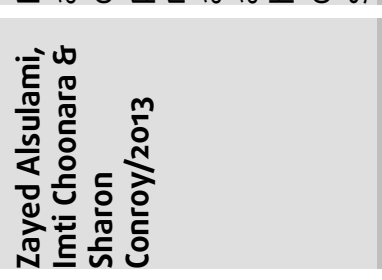 & 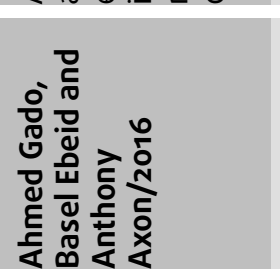 & 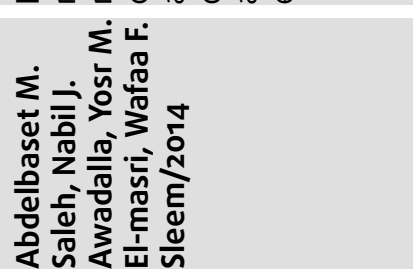 \\
\hline
\end{tabular}




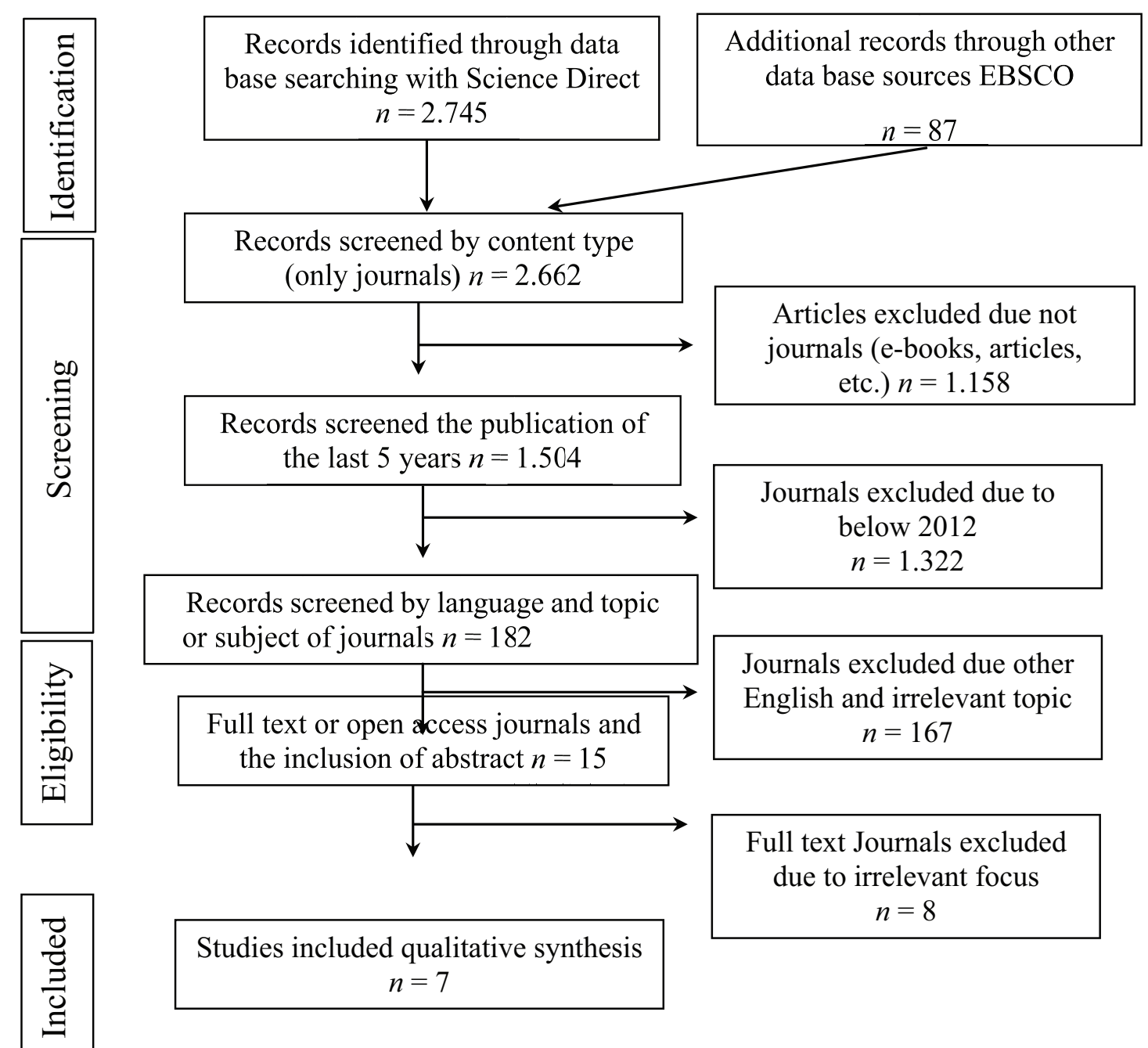

Figure 1: PRISMA diagram demonstrating the search strategy and its results.

\subsection{Dose errors}

The study by Saaghafi and Zargarzadeh in two largest hospital of Isfahan, Iran reported a total number of recorded data were 8,162 , from which 8,000 were complete and used for analysis. This data belonged to 565 patients and was collected in 4 stages. The number of records in each stage was 1,000 in each hospital or 2,000 records for each of the four stages. Administration error was $41.5 \%$ in Al-Zahra Hospital and $34 \%$ in Dr. Shariati Hospital. On an average, the most frequent error was the wrong time (15.6 $\%$ ) and omission, unauthorized dose (4.7\%), under-dose (4.6\%), extra dose $4.5 \%$ ), wrong route $(3.3 \%)$, and wrong frequency (0.5\%). For example, wrong route error means when an intravenous drug was administered subcutaneously. Wrong time error 
occurred when a drug was administered $>1$ hour before or after the scheduled time. The above mentioned process went on until the records of 1,000 doses were collected in each of the two hospitals. A great portion of errors were wrong time errors, none of the cases led to fatality. They found more than a single administration error for almost $1 \%$ of all 2000 doses [5].

\subsection{Time errors and route errors}

A research conducted by Saleh et al. in Mansoura Teaching University Hospital was reported 'wrong time' and 'wrong route' were the most frequent medication errors (23.1\% each). There were significant relationships between sleep medication intake and medication errors, depression, and fatigue. Linear regression analysis demonstrated that the independent predictors of medication errors were the number of patients under the nurse's direct care and the depression score of the nurses [6].

\subsection{Drug errors}

Other study by Kim and Bates at Korea University Teaching Hospital reported 45.6\% of nurses verified the drug to be administered to patient at least just one second). In addition, only $6.5 \%$ read the name of the patient from the wristband. Administering the medication at the correct time guideline was observed $41.0 \%$ of the time [3].

The study by Alsulami, Choonara and Conroy in UK Hospital reported about total of 191 MEs detected during the study observation period, giving a MEs rate of $96 \%$ of drug administrations. The most frequent type of $M E$ is administering drugs for children that was given by their parents when the nurse was not present. There were 64 instances where this occurred. The nurse did not observe the administration of the drug by the parent. That is a deviation from the hospital policy and procedure for drug administration. The other errors identified included incorrect administration of the medicine [7].

The present of case report in Bolak Eldakror Hospital Egypt by Gado, Ebeid, and Axon, which accidental Intravenous administration of epinephrine instead of midazolam at colonoscopy, which epinephrine was accidentally administered intravenously instead of midazolam at colonoscopy. The error was due to problem with medicine storage, misplaced medication, misreading a medication label and wrong selection [8]. 


\subsection{Documentation errors}

The US Institute of Medicine (IOM) reported on human error and quality of care in 1999 stated that the majority of human errors were a result of systemic defects and a fundamental revision of the system is required in order to provide safe care. Ideally, systems should be designed so as to minimize the possibility of errors, and, should they occur, recognize errors in time to prevent consequences [9]. some data indicate that nurses and other health care staff may perceive medication errors differently [10].

Approximately $16 \%$ of nurses are not aware of the type of situations that lead to medication errors, and $14 \%$ do not know when to report an error. Nurses themselves believe that only $25 \%$ of all errors are reported on accidents sheets and only $3.5 \%$ believe that all medication errors must be reported [10, 11].

\section{Discussion}

According to the clinical risk management theory, risk identification is the first and fundamental step to manage as otherwise any strategy to reduce risk may be inappropriate [3]. Researchers used a direct observation approach to assess the medication error rate and found high rates of medication errors and non-adherence to guidelines, with violations occurring in about one of four items overall. The direct observation method identified the greatest number of drug-related problems (DRPs), while incident report review identified the fewest [12].

The observational method has shown to be objective and more reliable than other means of spontaneous reporting or patient chart reviews in detecting errors [13]. the most common type of error (64 errors) was related to drugs being given by the child's parents without observation by the nurses. This type of error can be considered a deviation from the hospital policy and procedures rather than an actual administration егror [14].

There are many reasons why medication errors occur [15]. It may be related to professional practice, products, procedures environment or systems; may involve prescribing and ordering; dispensing and distribution; preparation and administration; labelling, packaging, and nomenclature; communications and education; or use and monitoring of treatment. Commonly implication are medication factors (e.g., similar sounding names, low therapeutic index, polypharmacy), patient factors (e.g., impaired cognition, vision problems and other physical disabilities, poor renal or hepatic function) or health system-related factors (e.g., use of inappropriate abbreviations and 
decimal points on prescriptions, failure to read drug labels, overburdening, and fatigue of healthcare providers). In this case (labetalol injection had been given by mistake instead of spinal bupivacaine), the reasons were keeping similar looking ampoules together, failure on the part of the internship to read the label and failure on the part of the resident to cross-check that the correct medication was being injected [16].

The study by Hajibabaee et al. reported about findings showed that the mean number of reported medication errors during 3 months in the wards under study was 1.3 per nurse, whereas the mean number of medication errors that nurses recalled during the same period was 19.5. Most respondents reported making and reporting o errors. A small number made up to 40 errors of certain types and reported up to 20 . The mean number of reported medication errors of nurses who had participated in drug administration training courses less than that of the nurses who had not attended such training, although no significant difference was observed [9].

Two approaches to human error have been described: The person approach; and the systems approach and they are also applicable to the issue of medication errors [16].

The person approach focuses on the culpability of individuals. The tendency is to name, blame, and shame. The systems approach accepts that humans are fallible, and therefore, errors may occur regardless of the competence of individuals working within the system. Rather than focusing on the individual, it focuses on the conditions under which individuals work and how those conditions can predispose to errors. It is now widely acknowledged that although professionals must take responsibility for their actions, blaming doctors, pharmacists, or nurses for errors does not encourage a culture of reporting or learning [17].

Nurse is one of the spearheads in the delivery of health services in hospitals. Nurses have a very important role because they are 24 hours with the patients. They are the main actors in administration drugs based on orders physician or treatment program. But for many reasons, nurses often gave drugs that they did not prepare themselves and rarely washed their hands before giving oral or external medications. Other types of errors such as wrong patient and wrong dose errors were much less frequent, but may have more potential for harm. These data suggest that both implementation of educational strategies, and tracking of performance will be helpful. In the longer term, technological solutions such as bar coding and implementation of electronic medication administration records may also be helpful [3].

The repost of a study, there was variation between paediatric nurses adherence to double-checking steps during medication administration. The most frequent type of 
administration errors or deviation from policy involved the medicine being given to the parents to administer to the child when the nurse was not present [7].

Therefore, patients and nurses must alter their attitude and follow the physician instructions or medication programs, it's all for their own safety to be assured and ensuring medicine identification should be correct. It is crucial to improve the hospital culture among the nurses that confirming the identity of patients in drug administration is a very important part of their duties. Another more technical solution is bar coding with links to the medication administration record, which can automate checks of right patient, right drug and right dosage [3].

Based on research reports in several countries (two largest hospital of Isfahan, Iran; University Teaching Hospital, Korea; Mansoura Teaching University Hospital, case report in Bolak Eldakror Hospital, Egypt), medication errors should be categorized into five types of MEs: (1) Drugs error from 34 to $5.6 \%$; (2) Doses errors from 4.5 to 9.6 $\%$; (3) Route error $0.5 \%$, (4) Time error or frequency error from 15.6 to $23.1 \%$; and (5) Documentation error $30 \%$. Two case studies on the endoscopy unit in Eldakror Hospital, Egypt and the operating room in Midnapore Medical College and Hospital, India reported medication administration errors (drugs error) to patients.

\section{Conclusion}

Based on result of the studies, it could be concluded that medication errors are common throughout the hospital. There were many reasons why medication errors occurred. It may be related to professional practice, products, procedures, environment or systems [16].

The error was due to problem with medicine storage, misplaced medication, misreading a medication label and wrong selection. The error resulted in intervention (hospital admission and monitoring) but did not result in harm or death [8].

Based on the results of seven studies that have been done (table 1. Journal in review), author suggested that nurses have to improve understanding of the importance of medication administration. It is important for all nurses to become familiar with various strategies to prevent or reduce the likelihood of medication errors. Here are many strategies to help nurses do just that are: Ensure the six rights of medication administration principles (right patient, right medication, right dosage, right route of administration, right time and right documentation); Follow proper medication reconciliation procedures; Double check or even triple check procedures; Have the physician (or another nurse) read it back; Consider using a name alert; 
Document everything, ensure proper storage of medications for proper efficacy; Learn the institution's medication administration policies, regulations, and guidelines; and Consider having a drug guide available at all times.

\section{References}

[1] Kohn, L. T., Corrigan, J. M., and Donaldson, M. S. (2000). To Err is Human Building a Safer Health System.

[2] Stelfox, H. T., Palmisani, S., Scurlock, C., et al. (2006). The 'To Err is Human' report and the patient safety literature. Quality and Safety in Health Care, vol. 15, no. 3, Pp. $174-178$.

[3] Kim, J. and Bates, D. W. (2013). Medication administration errors by nurses: Adherence to guidelines. Journal of Clinical Nursing, vol. 22, no. 3-4, pp. 590-598.

[4] National Coordinating Council for Medication Error Reporting and Prevention. (2016). About Medication Errors. What is a Medication Error? NCCMERP. Retrieved from http://www.nccmerp.org/about-medication-errors

[5] Saghafi, F. and Zargarzadeh, A. H. (2014). Medication error detection in two major teaching hospitals: What are the types of errors? Journal of Research in Medical Sciences: The Official Journal of Isfahan University of Medical Sciences, vol. 19, no. 7, pp. 617-623.

[6] Saleh, A. M., Awadalla, N. J., El-masri, Y. M., et al. (2014). Impacts of nurses' circadian rhythm sleep disorders, fatigue, and depression on medication administration errors. Egyptian Journal of Chest Disease and Tuberculosis, vol. 63, no. 1, pp. 145-153.

[7] Alsulami, Z., Choonara, I., and Conroy, S. (2014). Paediatric nurses' adherence to the double-checking process during medication administration in a children's hospital: An observational study. Journal of Advanced Nursing, vol. 70, no. 6, pp. 1404-1413.

[8] Gado, A., Ebeid, B., and Axon, A. (2016). Accidental IV administration of epinephrine instead of midazolam at colonoscopy. Alexandria Journal of Medicine, vol. 52, no. 1, pp. 91-93.

[9] Hajibabaee, F., Joolaee, S., Peyravi, H., et al. (2014). Medication error reporting in Tehran: A survey. Journal of Nursing Management, vol. 22, no. 3, pp. 304-310.

[10] Koohestani, H. R. and Baghcheghi, N. (2008). Barriers to the reporting of medication administration errors among nursing students. Australian Journal of Advanced Nursing, vol. 27, no. 1.

[11] Osborne, J., Blais, K., and Hayes, J. S. (1999). Nurses' perceptions: When is it a medication error? Journal of Nursing Administration, vol. 29, no. 4, pp. 33-38. 
[12] Berdot, S., Sabatier, B., Gillaizeau, F., et al. (2012). Evaluation of drug administration errors in a teaching hospital. BMC Health Services Research, vol. 12, no. 1.

[13] Fortescue, E. B., et al. (2003). Prioritizing strategies for preventing medication errors and adverse drug events in pediatric inpatients. Pediatrics, vol. 111, no. 4, pp. 722729.

[14] Ghaleb, N., Abdullah Barber, M., Franklin, B. D., et al. (2009). The incidence and nature of prescribing and medication administration errors in paediatric inpatients. Archives of Disease in Childhood.

[15] Wakefield, B. J., Wakefield, D. S., and Uden-Holman, T. (2000). Improving medication administration error reporting systems. Why do errors occur? Ambulance Outreach, pp. 16-20.

[16] Laha, B. and Hazra, A. (2015). Medication error report: Intrathecal administration of labetalol during obstetric anesthesia. Indian Journal of Pharmacology.

[17] Benjamin, B.-0., et al. (2008). Medication errors and response bias: The tip of the iceberg. The Israel Medical Association Journal, vol. 10, no. 11, pp. 771-774. 